\title{
A Comparison between Cyclone Separator Efficiency Enhancement using Ferrous Powder and Additional Tangential Chamber
}

\author{
W. Mazyan; A. Ahmadi \\ School of Sustainable Design Engineering \\ University of Prince Edward Island \\ Charlottetown, Canada
}

\author{
M. Hoorfar \\ School of Engineering \\ University of British Columbia \\ Kelowna, Canada \\ mina.hoorfar@ubc.ca
}

\begin{abstract}
This paper provides a comparison between the separation efficiency in cyclones separators using two optimization techniques. Separation optimizations using ferrous powder, and additional tangential chamber were investigated. The proposed techniques show separation efficiency enhancements compared to the basic cyclone separator design. The separation ratio efficiency, $\varepsilon_{\mathrm{sp}}$, is evaluated by calculating the outlet to inlet count ratio. Similar to experimental studies in the literature, the inlet and outlet particle counts were evaluated using discretization techniques with the help of a microscope and a membrane collecting the dust at the outlet. It is observed from the two optimization techniques that the addition of ferrous powder while attracting it through magnetic forces provides an additional separation enhancement by $2 \%$ for $11 \mu \mathrm{m}$ particles sizes. This advantage comes with a total of $25 \%$ efficiency enhancement for $4 \mu \mathrm{m}$ particles compared to conventional designs. This study gives an insight on the different approaches proposed.
\end{abstract}

Keywords-component; cyclone separators; efficiency; ferrous powder; tangential chambers

\section{INTRODUCTION}

There has been an increasing demand for natural gas extraction in the last 50 years [1]. To meet this demand efficiently, there have been advances in all the natural gas processes. A special attention had been given to natural gas treatment process by which the natural gas is cleaned from residues and unwanted particles [2]. The efficiency enhancement in gas treatment leads to reduction in energy consumption, which in turns reduces the overall $\mathrm{CO}_{2}$ emissions into the environment.

The removal of solid and liquid particles is among the most important processes in natural gas treatment [2]. It is essential to remove the solid particles from the gas stream as they are considered to be the main reason in damaging the downstream equipment such as compressors, pressure vessels and heat exchangers. Many techniques have been innovated to remove these particles from the natural gas stream. Among the evolving technologies used to remove solid particles are the cyclone separators. Cyclone separators consist of a major barrel and a conical chamber by which the solid particles are separated from the main stream by centrifugal forces [3]. Compared to other solid-gas separators, the main drawback of cyclone separators would be its inability to separate small sized particles (i.e. particles smaller than $3 \mu \mathrm{m}$ at efficiencies higher than $75 \%$ ). On the other hand, cyclone separators have an advantage among other types of solid-gas separators (such as cartridge filters) and that is its low operating cost as well as its simple design [3].

Many researchers have focused on enhancing the cyclone separator efficiency. Among those, Kim et al. [4] proposed the introduction of guiding vanes to enhance the separation efficiency. Their results indicate an increase by $27 \%$ in separation efficiency for $4 \mu \mathrm{m}$ particles. Another study conducted by Lim at al. [5] investigated splitting the inlet of the cyclone separators into two parts. Their results showed a separation efficiency increase by $15 \%$ as compared to the conventional one inlet cyclone separator. In a similar study, Xiong et al. [6] attached vortex finders at the inlet of the cyclone separator. Their analysis showed that helical profile vortex finders could enhance the separation efficiency by $9 \%$ compared the straight profile vortex finders. Xiang et al. [7] also studied the effect of the conical chamber dimensions on the separation efficiency. Their study findings indicate that a decrease in the bottom radius of the cone from $19.4 \mathrm{~mm}$ to 11.6 $\mathrm{mm}$ would enhance the separation efficiency by $16 \%$. Yoshida et al. [8] suggested the study of the conical length effect on the separation efficiency. Their analysis showed that the $50 \%$ cut size was reduced. Park et al. [9] also investigated the effect of allocating cyclone separators in series with step-up cyclone chamber diameters. The first cyclone could segregate particle sizes ranging from $4.5-11 \mu \mathrm{m}$, the second cyclone could segregate particle sizes ranging from $3.4-7.9 \mu \mathrm{m}$, and the third cyclone could segregate particle sizes ranging from $1.8-$ $4.3 \mu \mathrm{m}$. The injection of mist was another proposed study analyzed by Yang and Yoshida [10]. In their analysis, they were able to achieve a $20 \%$ efficiency enhancement for $1 \mu \mathrm{m}$ particle size capturing at $20 \mathrm{~m} / \mathrm{s}$ inlet velocity.

Although many investigators have reached an enhancement in the separation efficiency, this study compares between two novel and simple techniques for separation efficiency enhancement. 


\section{Methodolgy}

In this study, a conventional cyclone separator based on Stairmand's design [11] was modified. Two modification techniques were applied. The first method studies the effect of adding ferrous powder along with magnets to enhance the separation efficiency. The magnetic force will act in the direction of the centrifugal force, and hence, separation efficiency will be enhanced. The second method studies the effect of adding a tangential collecting chamber at the conical section of the separator. As particle velocities increase in this section of the cyclone separator, the tangential collecting chamber will increase the tendency of capturing smaller solid particles at a higher rate, and hence, separation efficiency will be enhanced.

\section{A. Ferrous Powder Addition}

This novel technique involves mixing the gas-solid inlet mixture with adhesive ferrous powder. Particles entering with the gas flow get attached to ferrous powder through a helical mixing chamber. The solid particles will stick to the adhesive ferrous powder and then get attracted by permanent magnets attached to the walls of the cyclone separator in the major barrel [12]. The additional magnetic force is applied radially in the same direction as the centrifugal force; therefore, separation forces would increase and increase the separation efficiency. Figure 1 shows a schematic of the attraction process.

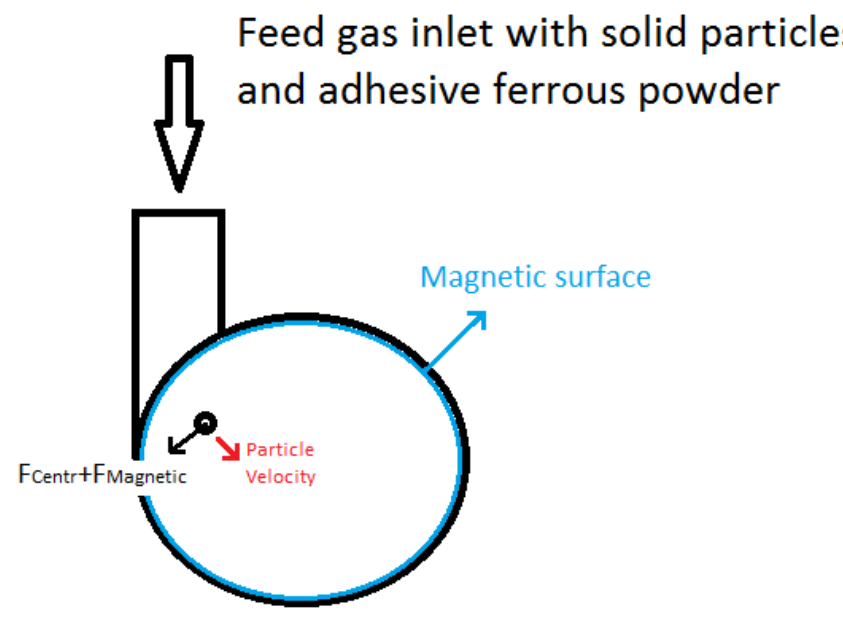

Figure 1: Schematic of ferrous powder addition

\section{B. Additional Tangential Chamber}

This novel technique suggests the use of an additional opening (collecting chamber) located at the conical section of the cyclone separator. The aim of the additional chamber is to collect particles at their highest angular velocities. Higher velocities conclude that the particles have higher tendency to reach outer walls of the conical section [13]. The additional collecting chamber at the conical section would increase the chances of collecting more particles reaching the walls of the conical chamber. This approach will reduce the possibility of escaping particles after bouncing off the conical chamber walls and escape with clean gas. Figure 2 shows an image of the additional tangential chamber located at the conical part of the cyclone separator.
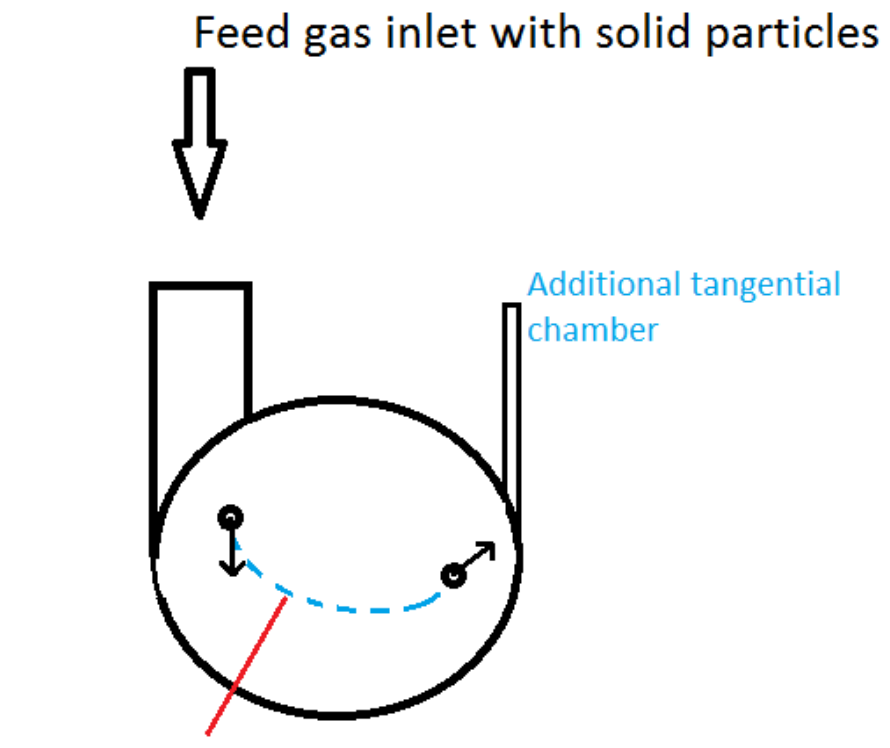

\section{Particle trajectory}

Figure 2: Additional conical tangential chamber

In both setups, the escaped solid particles are captured on a Whatman GE Healthcare membrane at the outlet stream. The exit outlet particles are also analyzed using the Qualitest Digital Microscope.

\section{ReSUlTS AND Discussions}

The separation efficiency evaluated in this study $\left(\varepsilon_{\mathrm{sp}}\right)$ is an indication of the cyclone separator performance. Therefore, higher values of the separation efficiencies indicate an enhanced cyclone separator and vice versa.

\section{A. The Effect of Ferrous Powder Addition}

Adding ferrous powder to the solid particles stream and recapturing the mixed (ferrous-solid) combination adds to the centrifugal force behaving on the solid particles in traditional cyclone separators. The additional force increases the chances of solid particles to reach the outer walls and get separated. Thus, the separation efficiency enhancement shown in Figure 3 explains the effect of the additional magnetic force compared to tradition cyclone separator performance.

\section{B. The effect of adding a tangential chamber}

Adding a tangential collection chamber at the conical section of the cyclone separator plays the role of capturing particles which reach the walls of the cone. This method raises the opportunity of capturing these particles while reducing the percentage of escaping particles with the fluid flow after hitting the walls. Thus, the separation efficiency enhancement shown in Figure 3 explains the effect of the additional tangential chamber compared to tradition cyclone separator performance

The separation efficiency results of both proposed techniques are shown in Figure 3. It is very clear that both techniques add to the separation efficiency. The minimum enhancement that both techniques provide is $25 \%$ at $4 \mu \mathrm{m}$ 
particles and $3 \%$ at $11 \mu \mathrm{m}$ particle sizes. Moreover, the separation efficiency enhancement using ferrous powder show better performance as compared to tangential chamber performance (i.e. ferrous powder technique shows a $2 \%$ higher separation performance compared to the tangential chamber addition). This enhancement could be due to a consistent force applied to all particles via the magnetic field. However, in the additional chamber process, not all particles will reach the outer walls of the cone at the chamber's opening. Therefore, the reduction of the tangential chamber performance compared to the ferrous powder is explained.

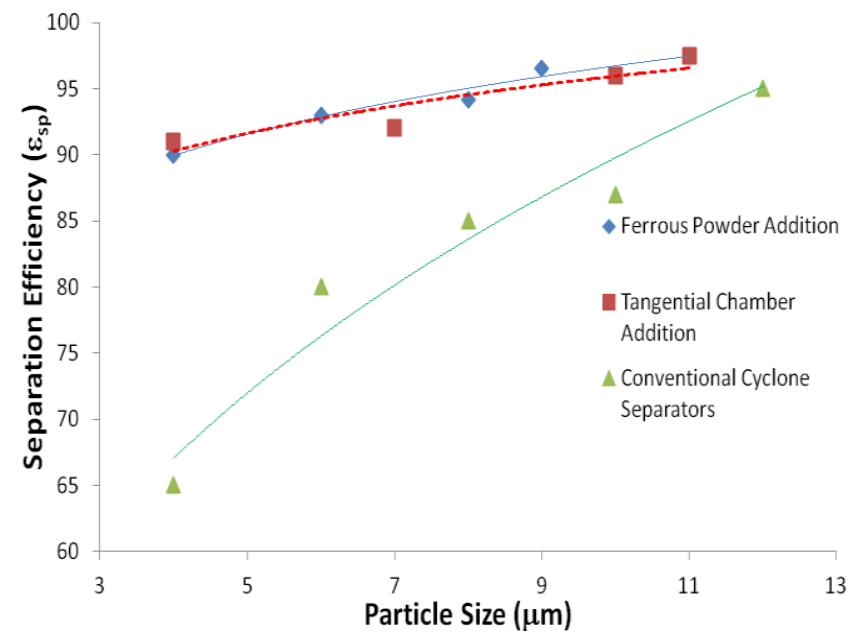

Figure 3: Comparison between proposed novel methods

\section{CONCLUSIONS}

The comparison between two novel techniques was investigated in this paper. Results show that these techniques, the ferrous powder addition and the tangential chamber addition enhance the separation efficiency of solid particles. On the other hand, the ferrous powder addition shows a better performance for larger particles as compared to the tangential chamber addition. Ferrous powder addition shows a $2 \%$ enhanced efficiency over the addition of the tangential chamber. This could represent the nature of the continuous attraction force exerted by the magnets on solid-ferrous particles combination. None the less, both approaches provide a minimum enhancement of at least $25 \%$ for $4 \mu \mathrm{m}$ particles. Thus, further research in the future could allow cyclone separators to have higher separation efficiencies and could allow cyclones separators to replace cartridge filters. Therefore, lower maintenance time and cost would be saved in the filtration industry.

\section{REFERENCES}

[1] M. Mei, "North American Natural Gas Market Dynamics. Canadian Energy Research Institute". Global LNG: A review study no. 123Section II, 2011.

[2] M. Scholes, G. Stevens, and S. Kentish, "Membrane gas separation applications in natural gas processing," Fuel, vol. 96, pp. 15-28, 2012.

[3] A. Bahadori, "Natural gas processing-technology and engineering design”. Waltham, MA: Elsevier inc, 2014.

[4] H. Kim, K. Lee, and M. Kuhlman, "Exploratory design modifications for enhancing cyclone performance," Aerosol Science, vol. 32, pp. 11351146, 2001.

[5] K. Lim, S. Kwon, and K. Lee, "Characteristics of the collection efficiency for a double inlet cyclone with clean air," Aerosol Science, vol. 34, pp. 1085-1095, 2003.

[6] Z. Xiong, Z. Ji, X. Wu, "Investigation on the Separation Performance of a Multicyclone Separator for Natural Gas Purification," Aerosol and Air Quality Research, vol. 14, pp. 1055-1065, 2014.

[7] R. Xiang, R. Park, S. Lee, and W. Lee, "Effects of cone dimension on cyclone performance," Journal of Aerosol Science, vol. 32, pp. 549-561, 2001.

[8] H. Yoshida, Y. Hayase, K. Fukui, T. Yamamoto, "Effect of conical length on separation performance of sub-micron particles by electrical hydro-cyclone," Powder Technology, vol. 219, 29-36, 2012.

[9] C. Park, D. Song, S. Yook, "Development of a single cyclone separator with three stages for size-selective sampling of particles," Journal of Aerosol Science,vol. 89, pp. 18-25, 2015.

[10] K. Yang, H. Yoshida, "Effect of mist injection position on particle separation performance of cyclone scrubber," Separation and Purification Technology, vol. 37 pp. 221-230, 2004.

[11] L. Brar, R. Sharma, K. Elsayed, "The effect of the cyclone length on the performance of Stairmand high-efficiency cyclone," Powder Technology, vol. 286, pp. 668:677, 2015.

[12] W. I. Mazyan, A. Ahmadi, R.D. Jesus, H. Ahmed and M. Hoorfar, "Use of ferrous powder for increasing the efficiency of solid particle filtration in natural gas cyclones," Separation Science and Technology, vol. 51, pp. 2098-2104.

[13] W. I. Mazyan, A. Ahmadi, H. Ahmed and M. Hoorfar, "Increasing efficiency of natural gas cyclones through addition of tangential chambers," Journal of Aerosol Science, vol. 110, pp. 36-42, 2017. 